\title{
The Improved Radial Source Recognition Algorithm Based on Fractal Theory and Neural Network Theory
}

\author{
Jinfeng Pang, Yun Lin* and Xiaochun Xu \\ College of Information and Communication Engineering \\ Harbin Engineering University, Harbin, China \\ linyun@hrbeu.edu.cn
}

\begin{abstract}
Nowadays, the traditional parameters recognition method cannot match the pequirements of the increasing new modulation radar signals. In order to solve this problem, in this paper, it proposes the improved radar signal recognition algorithm based on ractal theory and Neural Network theory. Taking the advantage of the characteristics of relevant dimension which will be able to measure the relevant comples degree of the radial source signals, we extract the relevant point as the input of neutral network in orden to recognize and classify the signals. Simulation results show that, this algorithm has a distinguish effect on classification under low SNR, which is suitable for the feature extraction and recognition of various styles of radar signals.
\end{abstract}

Keywords: Signal Recognition; Feature Extraction, Fractal Theory; Neural Network

\section{Introduction}

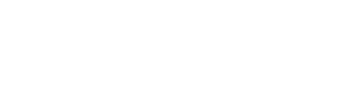

Radio signal recognition [1] plays an extremely crucial role in contemporary radar count measure, which has a cardintal step named feature extraction [2] and classifier design [3]. As the development of new style radar signal and complex of radar signal electromagnetism environment, radar signal recognition based on traditional feature parameters [4] would not afford the request, and a much more careful and effective feature is need for recognition.

Because traditionalmethod [5] is always making utilization of external parameter of radar signal as feature parameter, so the researchers can only recognize regular radar radiation source signal and those radiation source signals [6] whose feature parameter exist as constants, and have a low successful recognition rate and even it useless to new style radar signals. Fractal theory [7] has been widely used in the fields like seismic wave defection and image processing, which can quantificationally describe the complex and irregular degree of signals. Hausdorff Dimension is a basic fractal dimension in the theory; however, as a result, it brings out difficulties in the calculation. So we often utilize Box Dimension as a method to measure the complex degree of signals. Compared with Box Dimension, relevant dimension can calculate the relevant of different sample points of signals' internal in order to measure the complex degree of signals. And what is more, relevant dimension is able to extract the character of radar signals much more accurately. After effective feature extraction of signals, the design of classifier is surely to have a direct influence on signal recognition effect. Up to now, main kind of classifier has been near neighbor classifier, Fuzzy Logic classifier and Support Vector Machines classifier and so on, and Decision Tree classifier and 
Neutral Net classifier is the commonest. Result from the advantage of high robustness, fault tolerance and adaptive to environmental change itself, NN classifier has been widely utilized.

This paper has extracted 4 kinds relevant dimension feature of radar signals and used Neutral Net classifier-BP Net to train the extracted feature in order to achieve the destination of recognition of various styles of radar signals.

\section{Relevant Dimension Theory}

Relevant dimension is an exception of multiple fractal dimensions. Here we would introduce the relevant dimension simply from another aspect-multiple fractal dimension. Multiple dimensions describe the feature of different layers of substance and discuss the statistical distribution characteristic of parameters. We cut the research subject into $\mathrm{N}$ areas(taking the linear degree as 1), and suppose the linear degree of the area $i$ as $e_{i}$, then the density distribution function $P_{i}$ of area $i$ could be described in different scaling components as: $P_{i}=e_{i}^{\alpha_{i}}, i=1,2, \cdots, N_{i}$.

Non-integral is commonly called singular exponent, whose value is concerned with the area.

To get the distribution characteristic of series subsets, we define the function $X_{q}(e)$ to get the weight sum of every areas probability: $X_{q}(e)=\sum_{i=1}^{N} P_{i}^{q}$.

And we define general fractal dimension $D_{q}$ as.

$$
D_{g}=\frac{1}{q-1} \lim _{e \rightarrow 0} \frac{\ln X_{q}(e)}{\ln e}=\frac{1}{q-1} \lim _{e \rightarrow 0} \frac{\ln \left(\sum_{i=1}^{N} P_{i}^{q}\right)}{\ln e}
$$

We can get the relêvant dimension when $q$ equals to 2 .

\section{Neutra/Network Theory}

The BP Neural Network [12,13] is one of the most important and popular techniques in the field of Neuran Network, it is also a kind of supervised learning neural network, the principle behind which involves using the steepest gradient descent method to get any small approximation. A general model of the BP is shown in Figure 1.

In Figure 1, there are three layers contained in BP Neural Network (BPNN): input layer, hidden Tayer, and output layer. Two nodes of each adjacent layer are directly connected which is also called as a link. Each link has a weighted value presenting the relational degree between two nodes. Assume that there are $n$ input neurons and using a training process described by the following equations to update these weighted values, which can be divided into two steps:

(1) Hidden layer stage

The outputs of all neurons in the hidden layer can be calculated by following steps:

$$
\text { net }_{j}=\sum_{i=0}^{n} v_{i j} x_{i} \quad j=1,2, \cdots, m
$$




$$
y_{j}=f_{H}\left(\text { net }_{j}\right) \quad j=1,2, \cdots, m
$$

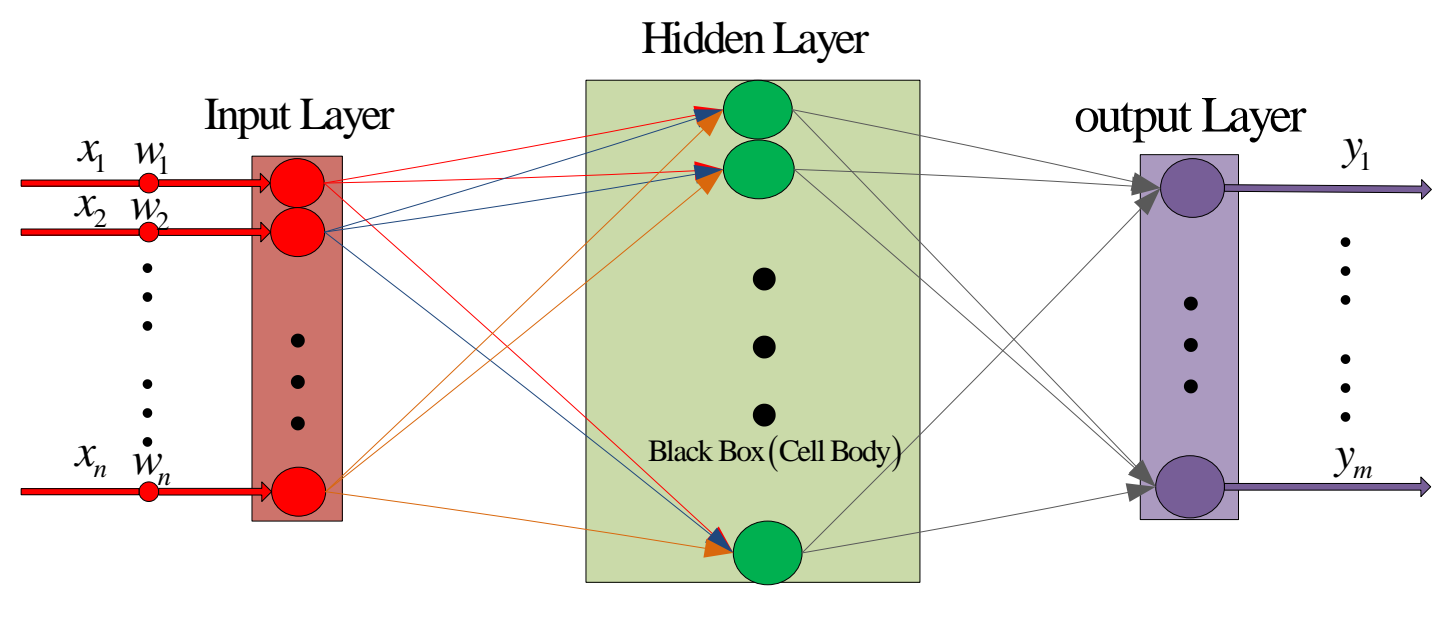

Here net ${ }_{j}$ is the activation value of the $j$ th rode, $y_{j}$ is the output of the hidden layer, and $f_{H}$ is called as the activation function of a node, usually a sigmoid function can be described as follows:

(2) Output layer stage

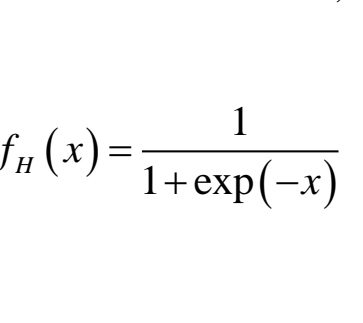

The outputs of all neurons in the output layer can be calculated as follows:

$$
\text { Dutput }=f_{\mathrm{O}}\left(\sum_{j=0}^{m} \omega_{j k} y_{j}\right)
$$

Here $f_{\mathrm{O}}$ is the activation function, which is usually a line function. All weights are assigned with initiallorandom values, and are modified by the delta rule according to the learning samples data.

\section{Simulation Experiment and Analysis}

Here we take 4 kinds radar signals which are FSK, PSK, LFM and CSF as signals for recognition, extract their relevant dimension characters and use the difference in the complex degree of different signals in order to recognize these 4 signals.

In the Figure 2, we show the relevance curves of relevant dimension $\ln e \square \ln X_{q}$ of 4 signals when the $\mathrm{SNR}$ is $0 \mathrm{~dB} 0 \mathrm{~dB}$ and $15 \mathrm{~dB}$ separately. 


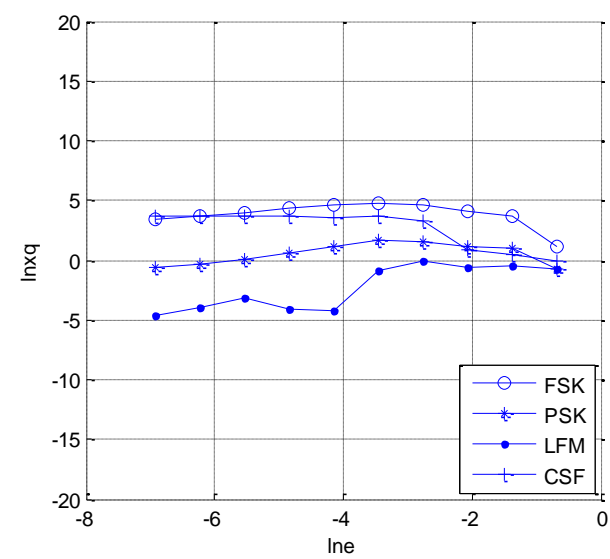

(a) $\mathrm{q}=2 \quad(\mathrm{SNR}=0 \mathrm{~dB})$

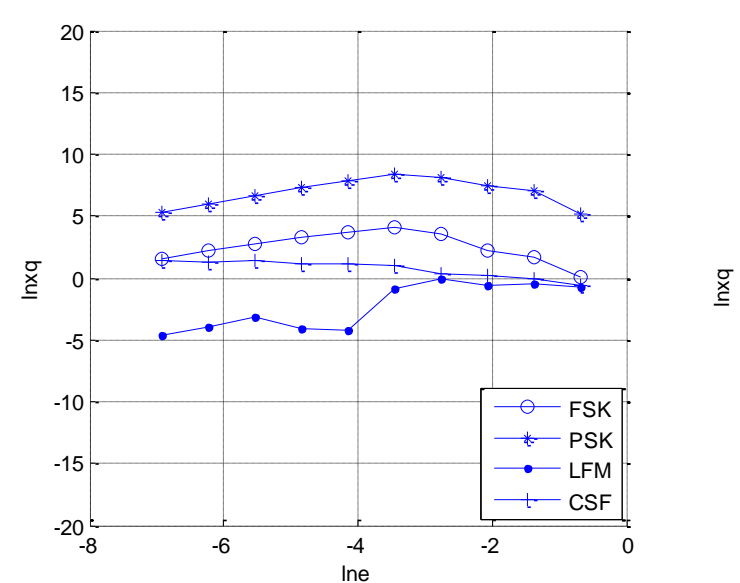

(c) $q=2(S N R=10 \mathrm{~dB})$

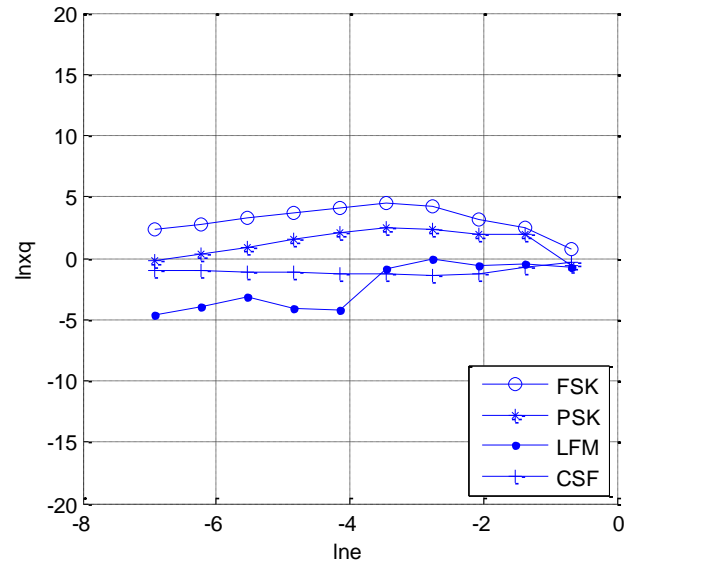

(b) $\mathrm{q}=2 \quad(\mathrm{SNR}=5 \mathrm{~dB})$

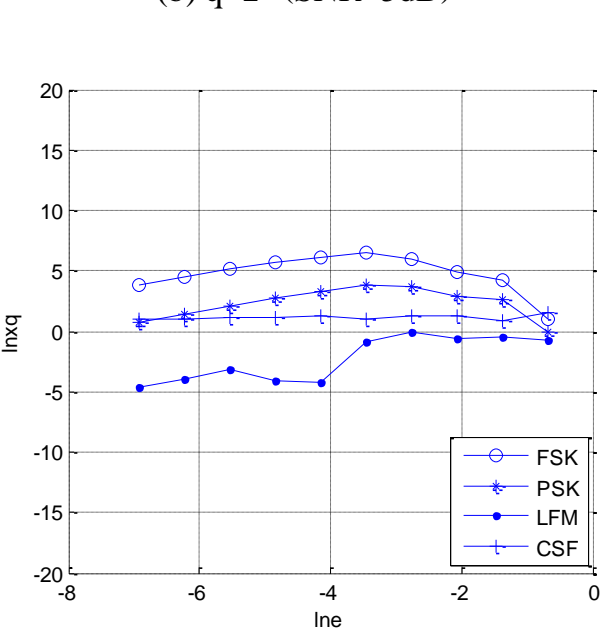

(d) $\mathrm{q}=2 \quad(\mathrm{SNR}=15 \mathrm{~dB})$

\section{Figure 2. Relevance character curves of 4 kinds of radar signals}

We can see from Figure 2 that there are some differences in relation curves of different signals, and the relevant feature is the value of $D_{2}$, according to $D_{q}=\frac{1}{q-1} \lim _{e \rightarrow 0} \frac{\ln X_{q}(e)}{\ln e}$ when $q$ is 2 . Since the relation curve of $\ln e \square \ln X_{q}$ which does not have a perfect linear relationship, it is certain to have a relatively large error when calculating the value of $D_{2}$. This experiment directly draws the relationship of $\ln X_{q}$ and $\ln e$ separately as features of different radar signals and input the neutral net in order to train for recognition. Then, each signal has got a feature vector as recognition feature aiming at extracting more carefully the difference between various signals, which would be fantastic evidence to the following work of neutral net work.

After extracting the relevant dimension of signals, we input the signals into neutral network which would train these signals and get successful recognition rate curve as shown in Figure 3. 


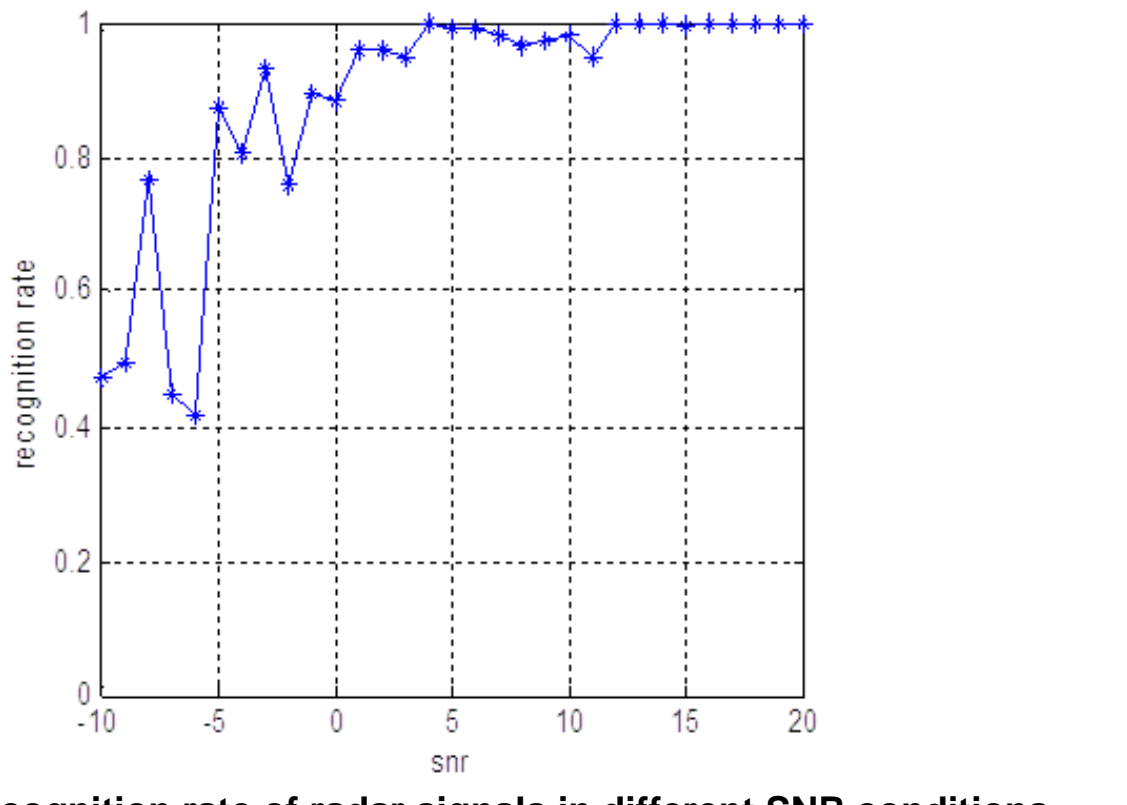

Figure 3. Recognition rate of radar signals in different SNR conditions

For these 4 radar signals, we extracted 100 samples of each kind and took 50 samples as training samples and the else as testing samples. Then we got 200 training and 200 testing samples as input of neutral network. The input layer of neutral network has 4 units which are accidently to be the number of output layers, and units of hiding layer are set to be 3 . The result in Figure 2 suggests that we have got a better recognition rate when SNR is larger than $-5 \mathrm{db}$, and even a perfect $100 \%$ recognition rate. So, it has been testified that this radar signal recognition algorithm has got a terrific effect on classification based on relevance curve feature and NNs.

\section{Conclusion}

This pape has been exhibiting a radar recognition algorithm based on relevance feature and neutral network. Since the relevant feature extracted has a poor linear character, the paper gave out a new method to regard the points of relevance curves as feature vectors which are the input of neutrahnetwork. Simulation analysis shows that the method has got a perfect recognition effect and a high recognition rate when SNR is larger than $-5 \mathrm{~dB}$. Especially, this feature extracting method has a much more widely utilization in the circumstance of fickle radar style environment.

\section{Acknowledgements}

This work is supported by the Nation Nature Science Foundation of China No.61301095 and 61201237, Nature Science Foundation of Heilongjiang Province of China NO. QC2012C069 and the Fundamental Research Funds for the Central Universities No. HEUCFZ1129, NO.HEUCF130810 and No. HEUCF130817.

\section{References}

[1] F. Brännström, Rasmussen and K. Lars, "Classification of Unique Mappings for 8PSK Based on Bit-wise Distance Spectra", Transactions on Information Theory, vol. 55, no. 3, (2009), pp. 1131-1145. 
[2] M. Ren, J. Cai, Y. Zhu and J. Han, "Radar Signal Feature Extraction Based on Wavelet Ridge and High Order Spectra Analysis", IET International Radar Conference, (2009) April 20-22; Guilin, China.

[3] S. Liaqat, S. A. Khan, M. B. Ihsan, S. Z. Asghar, A. Ejaz and A. I. Bhatti, "Automatic Recognition of Ground Radar Targets Based on Target RCS and Short Time Spectrum Variance", International Symposium on Innovations' in Intelligent Systems and Applications, (2011) April 15-18; Istanbul, Turkey.

[4] C. Ting and C. Wei, "A Fuzzy Pattern Recognition Method of Radar Signal Based on Neural Network", International Conference on Natural Computation, (2010) August 10-12; Yantai, China.

[5] X. Ma, X. Fang, R. Zhang and J. Xiang, "An Approach of Radar Clutter Recognition Based on Higher-order Statistics Combination”, (2000) August 21-25; Beijing, China.

[6] G. L. Avdeyenko, I. L. Lipchevskaya, Matyash, A. Yu and E. A. Yakornov, "The Phase Radio System for Coordinate Determination of a Radiation Source with a Phase Manipulation Signal located in the Fresnel region", Microwave and Telecommunication Technology International Crimean Conference, (2013), September 8-14, Sevastopol, Ukraine.

[7] X. Ying, L. Min, T. Bin, et al. "Feature-Extracting Method for Radar Compound Jamming Signal Based on Box Dimension and L-Z Complexity", Journal of Data Acquisition and Processing, vol. 23, no. 6, (2008), pp. 663-667.

[8] S. Gunasekaran and K. Revathy, "Fractal dimension analysis of audio signals for Indian musical instrument recognition”, 2008 International Conference on Audio, Language and Image Processing, Proceedings, (2008) July 7-9, Shanghai, China.

[9] S. Xu, J. Yang, Y. Wang, H. Liu and J. Gao, "Application of Fractal Art tor the Package Decoration Design", Proceeding 2009 IEEE 10th International Conference on Computer-Aided Industrial Design and Conceptual Design: E-Business, Creative Design, Manufacturing, (2009) November 26-29; Wenzhou, China.

[10] K. Zarajabad and H. S. Nikmehr, "A Novel Fractal Geometry for Harmonic Suppression in Parallel Coupledline Microstrip band pass filter", 2008 International Conference on Mrowave and Millimeter Wave Technology Proceedings, (2008) April 21-24; Nanjing China.

[11] K. Ambos-Spies, W. Merkle, J. Reimann and F Stephan, "Hausdorf Dimension in Exponential Time", Proceedings of the Annual IEEE Conference on Computational Complexity, (2001) June 18-21, Chicago, IL, United States.

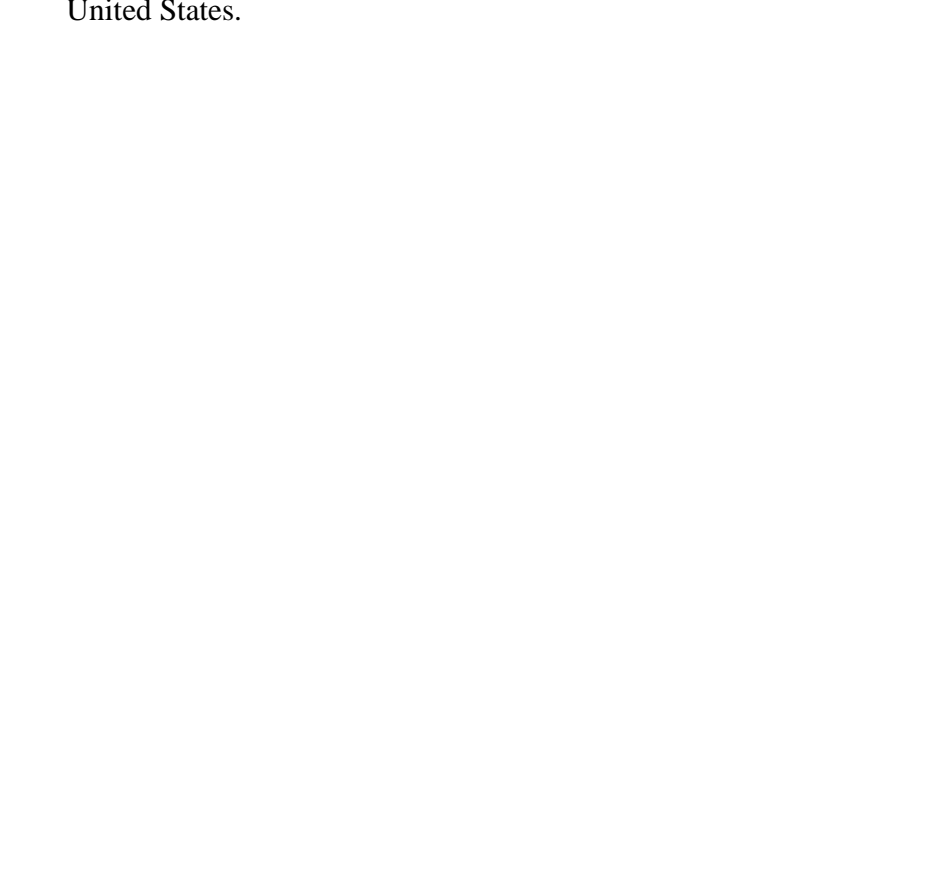

